\title{
The best quintic chebyshev approximation of circular arcs of order ten
}

\author{
Abedallah Rababah \\ Department of Mathematics, Jordan University of Science and Technology, Jordan
}

\begin{tabular}{|c|c|}
\hline Article Info & ABSTRACT \\
\hline Article history: & \multirow{6}{*}{$\begin{array}{l}\text { Mathematically, circles are represented by trigonometric parametric equations and im- } \\
\text { plicit equations. Both forms are not proper for computer applications and CAD sys- } \\
\text { tems. In this paper, a quintic polynomial approximation for a circular arc is presented. } \\
\text { This approximation is set so that the error function is of degree } 10 \text { rather than } 6 \text {; the } \\
\text { Chebyshev error function equioscillates } 11 \text { times rather than } 7 \text {; the approximation } \\
\text { order is } 10 \text { rather than } 6 \text {. The method approximates more than the full circle with } \\
\text { Chebyshev uniform error of } 1 / 2^{9} \text {. The examples show the competence and simplicity } \\
\text { of the proposed approximation, and that it can not be improved. }\end{array}$} \\
\hline Received Feb 19, 2019 & \\
\hline Revised Apr 4, 2019 & \\
\hline Accepted Apr 9, 2019 & \\
\hline Keywords: & \\
\hline Tich norformonocomputin & \\
\hline
\end{tabular}

Bézier curves

Quintic approximation

Circular arc

Copyright (C) 2019 Insitute of Advanced Engineeering and Science.

High accuracy

Approximation order

CAD

\section{Corresponding Author:}

Abedallah Rababah,

Department of Mathematics,

Jordan University of Science and Technology,

22110 Irbid, Jordan.

Email: rababah@just.edu.jo

\section{INTRODUCTION}

Only NURBS (Non Uniform Rational B-Splines) are capable to properly symbolize circles. NURBS have mathematical frame and harnessing them depends on mathematical knowledge and, in significant cases, demands the potential of employing geometric and analytic concepts. However, circles are directed to be used by people of limited and exclusive knowledge of mathematical notions and processes like computer graphics designers, stylers, animators, computer aided designers, and engineers. Drawing a circle in any CAD system is the primary alphabet in any software. So, it is very genuine to have the circle as a primitive and as a builtin-function in the software. A circle can be represented using rational Bézier curves and can be approximated by polynomial curves. Therefore, approximating a circular arc by polynomial curves with highest possible accuracy is a very important matter [1].

We treat the circular arc $c: t \mapsto(\cos (t), \sin (t)),-\theta \leq t \leq \theta$, see Figure 1 , to be approximated by a polynomial curve with superior uniform approximation. To come to this consequence, the geometric symmetries of the circle are used to fairly choose the Bézier points in order to symbolize the quintic Bézier curve that has highest approximation order of 10.

The circle $c$ is approximated in this paper using a quintic parametrically defined polynomial curve $p: t \mapsto(x(t), y(t)), 0 \leq t \leq 1$, where $x(t), y(t)$ are polynomials of degree 5 , that approximates $c$ with least deviation. Many researchers have tackled this issue using different degrees, norms, and methods, see for example $[2,3,4,5,6,7,8,9]$ and the references therein.

In [10], methods for approximating circular arcs using quintic polynomial curves with different boundary conditions are considered. The results of our method in this paper outperform the results in [10], 
are optimal, and can not be improved.

The error function to measure the distance between $p$ and $c$ is the following radial basis distance:

$$
E(t):=\sqrt{x^{2}(t)+y^{2}(t)}-1 .
$$

The radial distance formula contains radicals and thus will be replaced by the following deviation measure:

$$
e(t):=x^{2}(t)+y^{2}(t)-1 .
$$

Since the error function $e(t)=0$ represents the implicit equation of the circle; thus it is the suitable measure to test if $x(t)$ and $y(t)$ satisfy the equation of the circle and to measure the error.

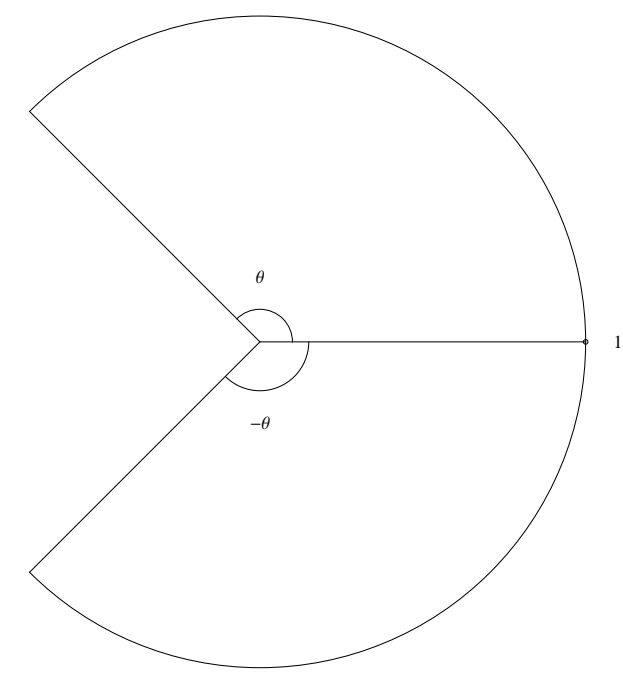

Figure 1. A circular arc

Among all possible kinds of approximations, the best uniform approximation yields the most accurate approximation. Unfortunately, in the literature, there is no method to find the best uniform approximation of degree five that approximates with order 6 [11]. In this paper, the quintic best uniform approximation is found that approximates with order 10 rather that 6 . This is a significant improvement over the order of the Chebyshev and Borel theorems [11]. In particular, the approximation problem that is considered in this paper is to locate a polynomial curve $p: t \mapsto(x(t), y(t)), 0 \leq t \leq 1$, where $x(t), y(t)$ are quintic polynomials, that "mimics" $c$ and minimizes $\max _{t \in[0,1]}|e(t)|$. Moreover, the following conditions are fulfilled:

(a) $e(t)$ equioscillates 11 times over $[0,1]$ rather than 7 ,

(b) $p$ approximates $c$ with order 10 rather than 6 .

These conditions are used to locate the Bézier points and to get the values of the parameters that are utilized to satisfy the geometric conditions of the circular arc. For more on these topics, see [1]. To achieve the conditions of the issue of the approximation, the following feature of the Chebyshev polynomials is used. Namely, the monic Chebyshev polynomial $\tilde{T}_{10}(u), u \in[-1,1]$, given by

$$
\tilde{T}_{10}(u)=\frac{-1}{512}+\frac{25}{256} u^{2}-\frac{25}{32} u^{4}+\frac{35}{16} u^{6}-\frac{5}{2} u^{8}+u^{10}, \quad u \in[-1,1]
$$

is the unique polynomial of degree 10 that equioscillates 11 times between $\pm \frac{1}{2^{9}}$ for all $u \in[-1,1]$ and has the least deviation from the $x$-axis [11].

Since the uniform error (for $e(t)$ ) equals $2^{-9}$, so, we allow the angle $\theta$ to be as large as possible in order to approximate the largest circular arc with this specified error. Thereafter, this angle $\theta$ has to be scaled by a factor that also combined with a reduction in the uniform error, see the last conclusions and open problems' section. 


\section{RESEARCH METHOD}

The curve $p(t)$ is given in Bézier form in order to use the Bézier technique [13]. See Figure 2 for possible Bézier points of a quintic Bézier curve. A Bézier curve $p(t)$ of degree 5 is given by

$$
p(t)=\sum_{i=0}^{5} p_{i} B_{i}^{5}(t)=:\left(\begin{array}{c}
x(t) \\
y(t)
\end{array}\right), \quad 0 \leq t \leq 1
$$

The points $p_{0}, p_{1}, p_{2}, p_{3}, p_{4}$ and $p_{5}$ are called the Bézier points, and the polynomials $B_{0}^{5}(t)=(1-t)^{5}, B_{1}^{5}(t)=$ $5 t(1-t)^{4}, B_{2}^{5}(t)=10 t^{2}(1-t)^{3}, B_{3}^{5}(t)=10 t^{3}(1-t)^{2}, B_{4}^{5}(t)=5 t^{4}(1-t)$ and $B_{5}^{5}(t)=t^{5}$ are the wellknown quintic Bernstein polynomials.

Since our purpose is to represent the arc with a polynomial curve with the least possible error, it is not substantial for the errors to take place at the endpoints or elsewhere; it is significant to ensure that this annoyance is as low as conceivable no matter where the error occurs. Our approach considers lessen the wrongdoing over all of the segment $[0,1]$. To explore the Bézier form approximation of a circular arc, a careful selection of locations of the Bézier points should be well-done. These locations are substantial to earn the convenient curve that redeems the approximation conditions. Based on the symmetry property of the circle, the right choice for the beginning control point $p_{0}$ should obey the following form: $p_{0}=\left(\alpha_{0} \cos (\theta), \beta_{0} \sin (\theta)\right)$, where values of $\alpha_{0}$ and $\beta_{0}$ could but should not be the same. Similarly, for symmetry reasoning, the valid option for the end control point $p_{5}$ is $p_{5}=\left(\alpha_{0} \cos (\theta),-\beta_{0} \sin (\theta)\right)$. Set $p_{1}=\left(a_{1}, b_{1}\right)$, then the point $p_{4}$ has to be selected to satisfy the form $p_{4}=\left(a_{1},-b_{1}\right)$. Set the point $p_{2}=\left(a_{2}, b_{2}\right)$, then the point $p_{3}$ has to be selected to satisfy the form $p_{3}=\left(a_{2},-b_{2}\right)$. Using the substitutions $a_{0}=\alpha_{0} \cos (\theta), b_{0}=\beta_{0} \sin (\theta)$, then the convenient choices for the Bézier points have to be, see Figure 2,

$$
p_{0}=\left(\begin{array}{c}
a_{0} \\
b_{0}
\end{array}\right), p_{1}=\left(\begin{array}{c}
a_{1} \\
b_{1}
\end{array}\right), p_{2}=\left(\begin{array}{c}
a_{2} \\
b_{2}
\end{array}\right), p_{3}=\left(\begin{array}{c}
a_{2} \\
-b_{2}
\end{array}\right), p_{4}=\left(\begin{array}{c}
a_{1} \\
-b_{1}
\end{array}\right), p_{5}=\left(\begin{array}{c}
a_{0} \\
-b_{0}
\end{array}\right) .
$$

It will be apparent that there are more than one solution; the consonant solution of best approximation begins in the second quadrant and ends in the fourth quadrant counter clockwise. Therefore, in order to have the Bézier curve $p$ begin in the second quadrant, go counter clockwise through fourth, third, first, second, and ends in the fourth quadrant as the circular arc $c$, the following stipulations should be satisfied:

$$
a_{0}, a_{1}, b_{1}, b_{2}<0, a_{2}, b_{0}>0 .
$$

Employ the Bézier points in (5) in the Bézier curve $p(t)$ in (4) to obtain:

$$
p(t)=\left(\begin{array}{c}
x(t) \\
y(t)
\end{array}\right)=\left(\begin{array}{c}
a_{0}\left(B_{0}^{5}(t)+B_{5}^{5}(t)\right)+a_{1}\left(B_{1}^{5}(t)+B_{4}^{5}(t)\right)+a_{2}\left(B_{2}^{5}(t)+B_{3}^{5}\right) \\
b_{0}\left(B_{0}^{5}(t)-B_{5}^{5}(t)\right)+b_{1}\left(B_{1}^{5}(t)-B_{4}^{5}(t)\right)+b_{2}\left(B_{2}^{5}(t)-B_{3}^{5}(t)\right)
\end{array}\right), 0 \leq t \leq 1 .
$$

The Bézier curve is settled by the 6 parameters $a_{0}, a_{1}, a_{2}, b_{0}, b_{1}, b_{2}$ [12]. These parameters are hired to get the best uniform approximation. We want to impose the conditions on the polynomial curve $p$; the polynomials $x(t)$ and $y(t)$ are substituted into $e(t)$. This leads to a polynomail of degree 10 that is solved using a computer algebra system. These proceedings are demonstrated in the next section.

\section{RESULTS AND ANALYSIS}

The values of $a_{0}, a_{1}, a_{2}, b_{0}, b_{1}, b_{2}$ that satisfy the conditions of the approximation problem are specified numerically and rounded fittingly in the following theorem.

Theorem 1: The Bézier curve (7) together with the Bézier points in (5) and the values of the parameters $a_{0}, a_{1}, a_{2}, b_{0}, b_{1}$, and $b_{2}$ given by

$$
\begin{gathered}
a_{0}=-0.978179455549407, a_{1}=-1.338058000836784, a_{2}=2.365283682287911, \\
b_{0}=0.21241016393227463, b_{1}=-1.6287837424896061, b_{2}=-2.4356328876380307
\end{gathered}
$$

achieves the following three conditions: $p$ minimizes the uniform norm of the error function $\max _{t \in[0,1]}|e(t)|$ and approximates $c$ with order 10, and the error function $e(t)$ equioscillates 11 times in $[0,1]$. 
The error functions fulfill:

$-\frac{1}{2^{9}} \leq e(t) \leq \frac{1}{2^{9}},-\frac{1}{2^{9}(2-\epsilon)} \leq E(t) \leq \frac{1}{2^{9}(2+\epsilon)}$, where $\epsilon=\max _{0 \leq t \leq 1}|E(t)| \approx 2^{-10}, \forall t \in[0,1]$.

Proof: We begin by considering the polynomials $x(t)$ and $y(t)$ in equation (7) and substituting them into the error function $e(t)$ in (2). Disposition the phrase and performing several simplifications gives the following equation:

$$
\begin{aligned}
e(t) & =\left(4 b_{0}^{2}-40 b_{0} b_{1}+100 b_{1}^{2}+80 b_{0} b_{2}-400 b_{1} b_{2}+400 b_{2}^{2}\right) t^{10} \\
& +\left(-20 b_{0}^{2}+200 b_{0} b_{1}-500 b_{1}^{2}-400 b_{0} b_{2}+2000 b_{1} b_{2}-2000 b_{2}^{2}\right) t^{9} \\
& +\left(25 a_{0}^{2}+150 a_{0} a_{1}+225 a_{1}^{2}-100 a_{0} a_{2}-300 a_{1} a_{2}+100 a_{2}^{2}+65 b_{0}^{2}-570 b_{0} b_{1}+1225 b_{1}^{2}\right. \\
& \left.+1060 b_{0} b_{2}-4500 b_{1} b_{2}+4100 b_{2}^{2}\right) t^{8} \\
& +\left(-100 a_{0}^{2}-600 a_{0} a_{1}-900 a_{1}^{2}+400 a_{0} a_{2}+1200 a_{1} a_{2}-400 a_{2}^{2}-140 b_{0}^{2}+1080 b_{0} b_{1}\right. \\
& \left.-1900 b_{1}^{2}-1840 b_{0} b_{2}+6000 b_{1} b_{2}-4400 b_{2}^{2}\right) t^{7} \\
& +\left(200 a_{0}^{2}+1100 a_{0} a_{1}+1500 a_{1}^{2}-700 a_{0} a_{2}-1900 a_{1} a_{2}+600 a_{2}^{2}+220 b_{0}^{2}-1420 b_{0} b_{1}+2000 b_{1}^{2}\right. \\
& \left.+2100 b_{0} b_{2}-5100 b_{1} b_{2}+2600 b_{2}^{2}\right) t^{6} \\
& +\left(-250 a_{0}^{2}-1200 a_{0} a_{1}-1350 a_{1}^{2}+700 a_{0} a_{2}+1500 a_{1} a_{2}-400 a_{2}^{2}-254 b_{0}^{2}+1320 b_{0} b_{1}\right. \\
& \left.-1450 b_{1}^{2}-1540 b_{0} b_{2}+2700 b_{1} b_{2}-800 b_{2}^{2}\right) t^{5} \\
+ & \left(210 a_{0}^{2}+830 a_{0} a_{1}+700 a_{1}^{2}-420 a_{0} a_{2}-600 a_{1} a_{2}+100 a_{2}^{2}+210 b_{0}^{2}-850 b_{0} b_{1}+700 b_{1}^{2}\right. \\
+ & \left.700 b_{0} b_{2}-800 b_{1} b_{2}+100 b_{2}^{2}\right) t^{4} \\
+ & \left(-120 a_{0}^{2}-360 a_{0} a_{1}-200 a_{1}^{2}+140 a_{0} a_{2}+100 a_{1} a_{2}-120 b_{0}^{2}+360 b_{0} b_{1}-200 b_{1}^{2}-180 b_{0} b_{2}\right. \\
+ & \left.100 b_{1} b_{2}\right) t^{3} \\
+ & \left(45 a_{0}^{2}+90 a_{0} a_{1}+25 a_{1}^{2}-20 a_{0} a_{2}+45 b_{0}^{2}-90 b_{0} b_{1}+25 b_{1}^{2}+20 b_{0} b_{2}\right) t^{2} \\
+ & \left(-10 a_{0}^{2}-10 a_{0} a_{1}-10 b_{0}^{2}+10 b_{0} b_{1}\right) t+\left(a_{0}^{2}+b_{0}^{2}-1\right) .
\end{aligned}
$$

The approximation conditions are satisfied if the error function is equalized with the polynomial of least deviation among all monic polynomials of degree 10 . So, the last equation which exemplifies the error function has to be equalized with the Chebyshev polynomial of first kind of degree $10, \tilde{T}_{10}(2 t-1) / 512$. We know that $\tilde{T}_{10}(u)=\cos (10 \arccos (u)), u \in[-1,1]$ is the unique monic polynomial of degree 10 that has the least deviation. It is given by equation (3), see [11]. Comparing the coefficients of equal powers of both sides and using the utilities of the computer algebra system in Mathematica, the solution that fulfills the conditions in (6) is established. Unfortunately, the solution is a collection of lengthy fractions and radicals that is impractical to write down the values of the parameters in this paper, so, we write them in decimal forms in equations (8) and (9). This shows that $p$ fulfills the three conditions of the approximation problem. To prove the error formula for $E(t)$, the relation to $e(t)$ is established. The error function $e(t)$ minimized is linked to the radial error $E(t)$ by the formulation:

$$
e(t)=x^{2}(t)+y^{2}(t)-1=\left(\sqrt{x^{2}(t)+y^{2}(t)}+1\right)\left(\sqrt{x^{2}(t)+y^{2}(t)}-1\right)=(2+E(t)) E(t) .
$$

Thus

$$
E(t)=\frac{e(t)}{2+E(t)}
$$

Substituting the bounds for $e(t)$ gives

$$
-\frac{1}{2^{9}(2-\epsilon)} \leq E(t) \leq \frac{1}{2^{9}(2+\epsilon)}, \text { where } \epsilon=\max _{0 \leq t \leq 1}|E(t)| \approx 2^{-10}, \quad t \in[0,1] .
$$

This completes the proof of Theorem 1 . 
The circular arc and the approximating Bézier curve are plotted in Figure 2. The resulting error between the curve and the approximation is not identified by the human eye which is clear from figure of the corresponding error plotted in Figure 3.

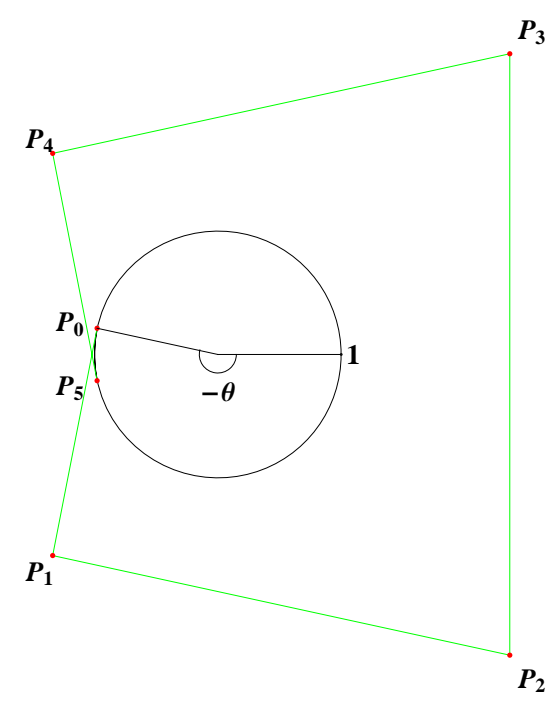

Figure 2. Circular arc and its quintic Bézier curve in Theorem 1.

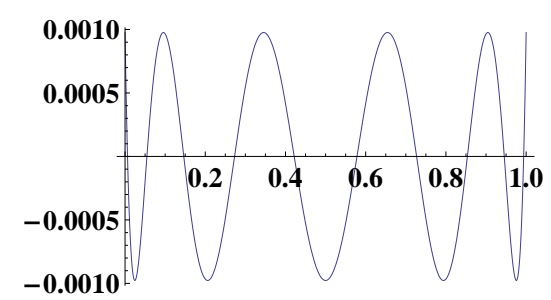

Figure 3. Radial Error of the quintic Bézier curve in Theorem 1.

The resulting Bézier curve reveals a brilliant positioning of the Bézier points to embrace more than a whole circle whilst possessing the Chebyshev error. We could not foresee a quintic polynomial to approximate more than the full circle further accurately than this approximation.

\section{CONCLUSION}

In this article, quintic approximation of the circle is established. The approximation fulfills extremely conclusive circumstances. Unlike the classical approximation that awards order of approximation of 6 , this approximation has order of approximation of 10; this is an superb acquisition. Moreover, in the significance of the Chebyshev norm, this approximation is the best and can not be improved. The error function equioscillates 11 times rather than 7 . The numerical examples reveal how efficient this method is. The approximation intersects the circular arc 10 times with maximum error $2^{-9}$ and thus outperforming any other approximation. Further research and investigations can be accomplished by considering the following issues:

(a) Finding the quintic best polynomial approximation for other curves that are widely used in the computer's applications like the hyperbolas and the spirals.

(b) Using the quintic best polynomial approximation in this paper to find the offset curve approximations of the Bézier curves.

(c) Finding the quintic best polynomial approximation with geometric conditions, $\mathrm{G}^{1}$ - and $\mathrm{G}^{2}$-conditions at the end points. 
(d) Applying the method in this paper to approximate the bit error rate (BER) expressions of the differential M-PSK modulation [14].

(e) Finding the quintic best polynomial approximation to shorter arcs of the circle that has an error reduced according to the length of the arc relative to the given approximation to more than the whole circle.

(f) Using the results in this paper to obtain the optimal delays between consecutive requests (DCR) in LoWPAN border routers to limit request send rates [15].

(g) Making a valid conjecture similar to the conjecture in $[16,17]$ that improves the results of Chebyshev's and Borel's theorems to approximate a curve with $2 n+1$ equioscillations rather than for functions with $n+2$ equioscillations. This is a novel break through and treasure trove in the field of approximation theory; however, this is a challenging issue and our today's knowledge is not rich enough to tackle it.

\section{ACKNOWLEDGEMENT}

The author owes thanks for the reviewers for invaluable suggestions to improve an earlier version of this paper. This research was funded by Jordan University of Science and Technology.

\section{REFERENCES}

[1] K. Höllig, J. Hörner: "Approximation and Modeling with B-Splines," SIAM, Titles in Applied Mathematics, vol. 132, 2013.

[2] Y. J. Ahn and C. Hoffmann, "Circle approximation using LN Bézier curves of even degree and its application," Journal of Mathematical Analysis and Applications, vol. 410 Issue 1, pp. 257-266, February 2014.

[3] P. Bézier, "The mathematical basis of the UNISURF CAD system," Butterworth-Heinemann Newton, MA, USA, ISBN 0-408-22175-5, 1986.

[4] J. Blinn, "How many ways can you draw a circle?," Computer Graphics and Applications, IEEE, vol. 7, Issue 8, pp. 39-44, 1987.

[5] C. de Boor., et al., "High accuracy geometric Hermite interpolation," Computer Aided Geometric Design, vol. 4, pp. 269-278, 1988.

[6] S. W. Kim and Y. J. Ahn, "Circle approximation by quartic $\mathrm{G}^{2}$ spline using alternation of error function," J. KSIAM, vol. 17, Issue 5, pp. 171-179, 2013.

[7] A. Rababah, "The Best Uniform Quadratic Approximation of Circular Arcs with High Accuracy," Open Mathematics, vol. 14, pp. 118-127, 2016. DOI: https://doi.org/10.1515/math-2016-0012

[8] A. Rababah, "The best uniform quintic approximation of circular arcs with high accuracy," 14th International Conference of Numerical Analysis and Applied Mathematics, Rhodes, Greece, AIP Conference Proceedings vol. 1863, Issue 1, 060005, 2017. https://doi.org/10.1063/1.4992219.

[9] A. Rababah, "The best uniform cubic approximation of circular arcs with high accuracy," Communications in Mathematics and Applications, vol. 7, Issue 1, pp. 37-46, 2016.

[10] L. Fang, "Circular arc approximation by quintic polynomial curves," Computer Aided Geometric Design, vol. 15, Issue 8, pp. 843-861, 1998.

[11] J. Rice, "The approximation of functions," 1: linear theory., Addison-Wesley, 1964.

[12] A. Rababah, "Best sextic approximation of circular arcs with thirteen equioscillations," Proceedings of the Jangjeon Mathematical Society, vol. 21, Issue 1, pp. 111-123, 2018. DOI: http://doi.org/10.17777/pjms2018.21.1.111

[13] G. Farin, "Curves and Surfaces for Computer Aided Geometric Design," Academic Press, Boston, 1997.

[14] S. Alabed, "Performance Analysis of Differential Beamforming in Decentralized Networks," International Journal of Electrical and Computer Engineering (IJECE), vol. 8 Issue 3, pp. 1692-1700, 2018. DOI: http://doi.org/10.11591/ijece.v8i3.pp1692-1700

[15] C. Suwannapong and C. Khunboa, "An Approximation Delay between Consecutive Requests for Congestion Control in Unicast CoAP-based Group Communication," International Journal of Electrical and Computer Engineering (IJECE), vol. 9, Issue 3, June 2019. DOI: http://doi.org/10.11591/ijece.v9i3.

[16] A. Rababah, "Approximation von Kurven mit Polynomen und Splines," Ph. Dissertation, Stuttgart Universität, West Germany, 1992.

[17] A. Rababah, "Taylor theorem for planar curves," Proceedings of the American Mathematical Society, vol. 119, Issue 3, pp. 803-810, 1993. 


\section{BIOGRAPHY OF AUTHORS}

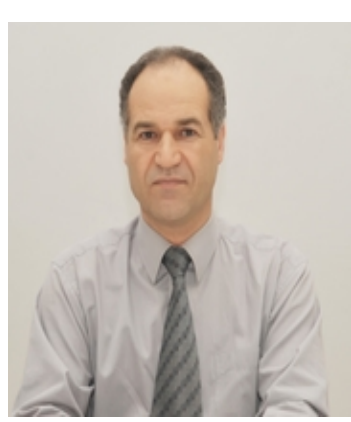

Abedallah Rababah is a professor of Mathematics at Jordan University of Science and Technology. He is working in the field of Computer Aided Geometric Design, abbreviated CAGD. In particular, his research is on degree raising and reduction of Bézier curves and surfaces with geometric boundary conditions, Bernstein polynomials, and their duality. He is known for his research in describing approximation methods that significantly improve the standard rates obtained by classical (local Taylor, Hermite) methods. He proved the following conjecture for a particular set of curves of nonzero measure: Conjecture: A smooth regular planar curve can, in general, be approximated by a polynomial curve of degree $\mathrm{n}$ with order $2 \mathrm{n}$. The method exploited the freedom in the choice of the parametrization and achieved the order $4 n / 3$, rather than $n+1$. Generalizations were also proved for space curves. Professor Rababah is also doing research in the fields of classical approximation theory, orthogonal polynomials, Jacobi-weighted orthogonal polynomials on triangular domains, and best uniform approximations. Since 1992, He has been teaching at German, Jordanian, American, Canadian, and Emirates' universities. He is active in the editorial boards of many journals in mathematics and computer science. Further info can be found on his homepage at ResearchGate: https://www.researchgate.net/profile/Abedallah_Rababah or at http://www.just.edu.jo/eportfolio/Pages/Default.aspx?email=rababah 